\title{
The Green Peas: Searching for LyC Emitters at Low Redshift
}

\author{
Anne Jaskot ${ }^{1}$ and Sally Oey ${ }^{2}$ \\ ${ }^{1}$ Dept. of Astronomy, Smith College, Northampton, MA 01063, USA \\ email: ajaskot@smith.edu \\ ${ }^{2}$ Dept. of Astronomy, University of Michigan, Ann Arbor, MI 48109, USA
}

\begin{abstract}
The escape fraction of Lyman continuum (LyC) radiation from galaxies remains one of the primary uncertainties in studies of reionization. However, few LyC-emitting galaxies are known. The recently identified, low-redshift "Green Pea" (GP) galaxies exhibit a number of similarities with high-redshift galaxies, and their optical emission lines suggest they may be some of the elusive LyC emitters. Recent HST COS and ACS observations of four GPs suggest further evidence for $\mathrm{LyC}$ escape and give new insights into the origins of $\mathrm{Ly} \alpha$ and low-ionization UV lines in high-redshift galaxies. The Ly $\alpha$ emission and low-ionization emission and absorption lines provide a coherent physical picture of the neutral gas distribution in the GPs and may identify LyC emitters at high redshift. The rare, low-redshift GPs hint at possible factors that may enable $\mathrm{LyC}$ and $\mathrm{Ly} \alpha$ escape from high-redshift galaxies.
\end{abstract}

Keywords. galaxies: evolution, galaxies: general, galaxies: high-redshift, galaxies: intergalactic medium, galaxies: ISM, galaxies: starburst, radiative transfer, ultraviolet: galaxies
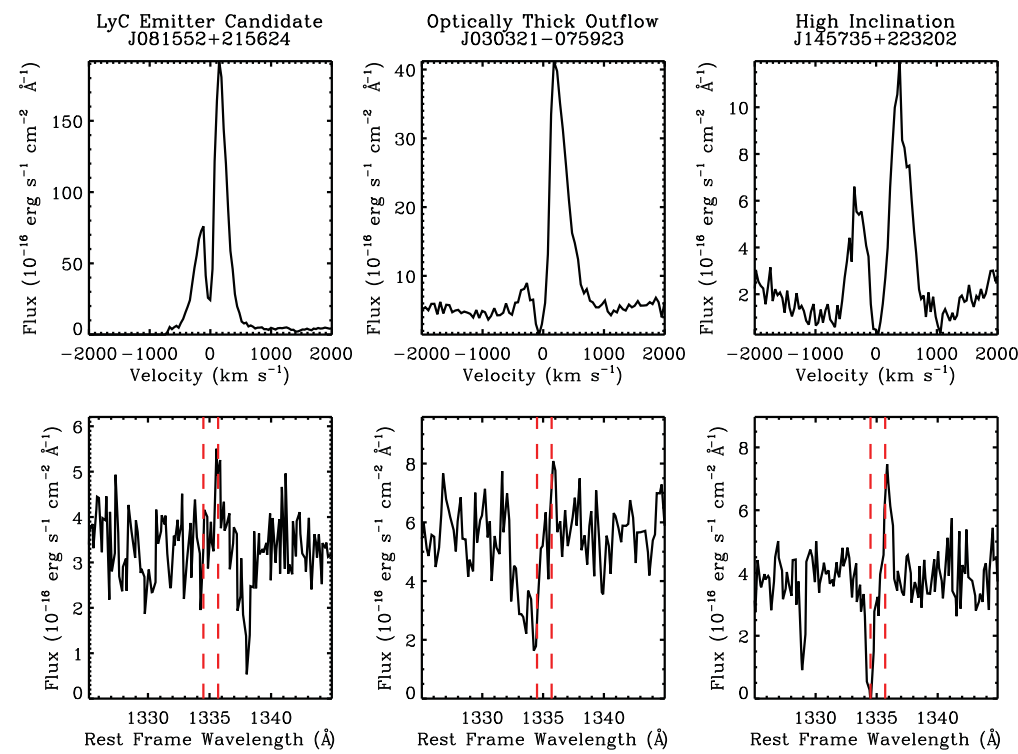

Figure 1. Top: Ly $\alpha$ emission from three Green Peas with different inferred optical depths and geometries. Bottom: The red lines show the expected positions of [C II] $\lambda 1334.5$ absorption and $\left[\mathrm{C}\right.$ II ${ }^{*} \lambda 1335.7$ emission. The $\mathrm{LyC}$ emitter candidates have strong, narrow $\mathrm{Ly} \alpha$, indicative of minimal scattering in neutral gas. They show scattered $[\mathrm{C} \mathrm{II}]^{*}$ emission but no line-of-sight $[\mathrm{C}$ II] absorption. The galaxy with an inferred optically thick outflow has deep, blue-shifted absorption and weaker, redshifted Ly $\alpha$ emission. The final Green Pea shows weak Ly $\alpha$ within an absorption trough and strong low-ionization absorption and emission from a high column density ISM. 